\title{
Changes in human sperm motion during capacitation in vitro
}

\author{
P. Morales, J. W. Overstreet, and D. F. Katz \\ Department of Obstetrics and Gynecology, Division of Reproductive Biology and Medicine, \\ University of California, Davis, California 95616, U.S.A.
}

\begin{abstract}
Summary. Spermatozoa from 10 fertile donors and from 10 patients with infertile marriages were washed and centrifuged (time zero, $\mathrm{T} 0$ ), and incubated in vitro in capacitation media for $6 \mathrm{~h}$ (T6), or $24 \mathrm{~h}$ (T24). At each time individual spermatozoa were classified as being morphologically normal or abnormal, and their movement characteristics were determined using high-speed videomicrography. Zona-free hamster oocytes were added to the T24 sperm suspensions. At all times, morphologically normal spermatozoa from donors and patients swam faster and had greater rolling frequency, flagellar beat frequency and amplitude than did abnormally shaped cells. Morphologically normal spermatozoa from donors exhibited a significant change in their movement pattern at $\mathrm{T} 6$. This change, which resembles hyperactivation in other species, was characterized by higher values of amplitude of lateral head displacement, and lower values of linearity, beat frequency and flagellar curvature ratio. In contrast, normal spermatozoa from patients showed only a decrease in straight line velocity at $\mathrm{T} 6$, with no other significant changes in movement characteristics. No changes in sperm movement could be demonstrated for the abnormal cells in either group of subjects. In sperm suspensions from donors and patients examined at T24, sperm vigour declined regardless of the morphological type. Spermatozoa from all 10 donors were able to penetrate the zona-free hamster oocytes, but spermatozoa from 5 of the 10 patients failed to penetrate oocytes. Correlations between hamster oocyte penetration and indicators of sperm vigour were demonstrated only for spermatozoa of patients.
\end{abstract}

Keywords: capacitation; sperm motility; hyperactivation; male fertility; sperm penetration assay

\section{Introduction}

In many mammals there are characteristic motility changes associated with sperm capacitation in vitro and in vivo. These changes are collectively termed hyperactivation (Yanagimachi, 1970; Fraser, 1977; Cooper et al., 1979; Cummins, 1982; Suarez et al., 1983; Katz et al., 1986; Behboodi et al., 1987; also see Yanagimachi, 1981). The most obvious expression of hyperactivation is a change in the pattern of sperm movement due to the increased bending of the proximal flagellum. The resulting trajectory is greatly curved and tortuous (Suarez et al., 1983; Katz et al., 1986). The functional significance of hyperactivation may involve the regulation of oviducal sperm transport and the generation of the forces required for sperm penetration of the granulosa cell vestments and the zona pellucida of the oocyte (Katz \& Yanagimachi, 1981; Yanagimachi, 1981; Katz et al., 1987). When hyperactivation of mammalian spermatozoa has been studied after capacitation in vivo and in vitro, the sperm movement characteristics in the two environments have been similar (Katz \& Overstreet, 1980; Katz \& Yanagimachi, 1980, 1981; Cummins, 1982).

Although hyperactivated motility is thought to be associated with human sperm capacitation, no detailed studies have been reported so far of the sperm flagellar movements under such 
conditions. Several investigators have made observations of alterations in human sperm swimming trajectories during in-vitro capacitation (Aitken et al., 1982, 1984, 1985; Burkman, 1984; Mortimer et al., 1984; Okada et al., 1985, 1986). These observations generally indicate a tendency for increasing curvature in the swimming trajectories (Burkman, 1984) and/or increased lateral motion of the sperm head along its path (Aitken et al., 1982; Mortimer et al., 1984). Attempts have been made to quantify these changes in the sperm trajectory in terms of an indicator called "amplitude of lateral head displacement" (David et al., 1981; Mortimer et al., 1984). Such descriptions are consistent with the patterns of hyperactivated motility observed in other mammals.

Hyperactivation has been described not only in terms of sperm swimming trajectories but also at the more fundamental level of flagellar movement characteristics (Katz et al., 1978, 1986; Suarez et al., 1983, 1984). No detailed observations have yet been published on the flagellar movements of hyperactivated human spermatozoa. In addition to its biological value, knowledge of these movements might be useful in the clinical assessment of human sperm function and male fertility.

In this study we have analysed the flagellar beat characteristics and the swimming trajectories of human spermatozoa under capacitating conditions. We have evaluated spermatozoa from fertile men and from patients being evaluated because of an infertile marriage. In an effort to associate abnormalities of sperm motion with abnormalities of capacitation, we also studied the interaction of these spermatozoa with zona-free hamster oocytes. Our previous results had indicated that morphologically abnormal spermatozoa may be dyskinetic, regardless of the fertility status of the donor (Overstreet et al., 1981; Katz et al., 1982; Morales et al., 1988). Therefore, we analysed separately, and then compared, the morphologically normal and abnormal cells for changes in flagellar movements and swimming trajectories that may be associated with capacitation.

\section{Materials and Methods}

Preparation of the spermatozoa. Single specimens from 10 fertile donors and from 10 patients undergoing fertility evaluation were collected by masturbation into sterile plastic containers and analysed within $1 \mathrm{~h}$ of ejaculation. The semen samples studied in these experiments were the same specimens described previously and the characteristics of these men and their ejaculates have been reported in detail (Morales et al., 1988). Semen was diluted with the culture medium of Biggers et al. (1971), as modified by Yanagimachi et al. (1979) and supplemented with 0.3\% bovine serum albumin (BSA, Sigma Chemical Co., Fraction V); this medium is referred to hereafter as BWW. The diluted semen was centrifuged at $500 \mathrm{~g}$ for $6 \mathrm{~min}$ in a $15 \mathrm{ml}$ polystyrene centrifuge tube (Corning Glass Works, Corning, NY); the sperm pellet was washed twice with Medium BWW, and the washed cells were then resuspended in $1 \mathrm{ml}$ Medium $\mathrm{BWW}$ for incubation in the tube at $37^{\circ} \mathrm{C}$ under $5 \% \mathrm{CO}_{2}$ in air.

High-speed video recording. Sperm suspensions were videotaped immediately after washing (time zero, T0) and after incubation in vitro for $6 \mathrm{~h}$ (T6) or $24 \mathrm{~h}$ (T24). Samples were prepared for videotaping by pipetting $20 \mu \mathrm{l}$ of the sperm suspension into a fiat capillary tube $200 \mu \mathrm{m}$ deep (Vitro Dynamics, Rockaway, NJ) to minimize sticking of the spermatozoa to glass. All the recordings were made using positive phase contrast optics with a $\times 20$ dry objective lens. An air curtain (Sage Instruments, Elk Grove Village, IL) maintained temperature at $37^{\circ} \mathrm{C}$.

Videotape recording was carried out at a rate of $60 \mathrm{fields} / \mathrm{sec}$ in $1 / 500 \mathrm{sec}$ exposures by a shuttered high-speed video camera (Tritronics, Burbank, CA). A record of elapsed time in $0.01 \mathrm{sec}$ intervals, generated by a video time generator (For A, Los Angeles, CA), was simultaneously recorded on a video cassette recorder (Victor Co., Tokyo, Japan). Details of these procedures have been published previously (Katz et al., 1986; Morales et al., 1988).

Videotape analysis. The videotapes were analysed manually frame by frame. Details of the analysis procedure have been given elsewhere (Katz et al., 1982; Morales et al., 1988). Using a transparent overlay imprinted with two rectangles corresponding to a length of $3-5 \mu \mathrm{m}$ and a width of $2-3 \mu \mathrm{m}$ respectively, 25 normal and 25 abnormal spermatozoa were identified in each ejaculate (Katz et al., 1982). Although spermatozoa were classified as normal or abnormal on the basis of their head morphology, $3 \%$ of the abnormal spermatozoa also had abnormal flagella. Each spermatozoon was analysed for straight-line velocity (VSL), which was measured as the straight-line distance traversed by the head-midpiece junction over $1 \mathrm{sec}$. The linearity (LIN) was the ratio of the straight line distance to the total curvilinear distance traversed by the head-midpiece junction over 1 sec. Higher values of LIN denote a straighter swimming trajectory. Rolling frequency (RF) of the sperm head was measured in rolls/sec. Amplitude of lateral head displacement $(\mathrm{AH})$ was determined by measuring the average deviations of the sperm head from the line of progression (David et al., 1981; Mortimer et al., 1984). Larger AH values indicate greater yawing by the sperm head. Flagellar beat frequency (BF) was counted during the interval required for 10 beats. Curvature ratio (CR) was 
calculated for the flagellar contraction exhibiting maximum midpiece curvature. CR was defined as the straight-line distance from the head-midpiece junction to the first geometric inflection point of the tail, divided by the curvilinear distance between these two points as measured along the tail (Suarez et al., 1983). The lower the value of CR, the greater is the bending in the proximal flagellum. The flagellar shape was traced at the point in a beat cycle where the distal fiagellum exhibited maximal bending, and the flagellar amplitude (AMP) was determined (Katz et al., 1982).

Sperm penetration assay. Oocytes were recovered from superovulated female golden hamsters (Yanagimachi $e t$ al., 1976). Cumuli and zonae were removed with $0.1 \%$ hyaluronidase (Sigma Chemical Co.) and $0.1 \%$ trypsin (Sigma) respectively, in Medium BWW, followed by rinsing in Medium BWW. A $0.15 \mathrm{ml}$ sample of the T24 sperm suspension was placed under mineral oil in a plastic Petri dish and 30 or more zona-free oocytes were introduced into the sperm suspension. The concentration of the sperm suspension had been adjusted to $10 \times 10^{6}$ motile spermatozoa $/ \mathrm{ml}$. The preparations were incubated at $37^{\circ} \mathrm{C}$ with $5 \% \mathrm{CO}_{2}$. After $3 \mathrm{~h}$ the oocytes were removed and rinsed in Medium BWW and then evaluated for the presence of swollen sperm heads using phase-contrast microscopy.

Statistics. Student's paired $t$ test was used to compare the mean values of the movement characteristics for the morphologically normal and abnormal spermatozoa, and also to compare the same morphological types at different times during in-vitro incubation. To study the correlation of sperm movement characteristics with the success of hamster oocyte penetration, the Spearman rank correlation test was applied.

\section{Results}

Comparison between the movements of morphologically normal and abnormal spermatozoa during invitro incubation

The values of the 7 movement characteristics measured at 3 times during incubation are shown for the fertile donors and for the patients in Table 1. The results show that the morphologically normal spermatozoa swam faster than the abnormal spermatozoa at all the times examined. Normal spermatozoa also had higher values of RF, flagellar BF and flagellar AMP than the abnormally shaped cells at all times. These differences between morphologically normal and abnormal spermatozoa were apparent in donors and patients. The values of LIN, AH and CR were not different between the morphologically normal and abnormal spermatozoa from the donors at $\mathrm{T} 0$ or for the patients at T0 and T6 (Table 1). However, at T6 morphologically normal cells from the donors had higher values of $\mathrm{AH}$ and lower values of LIN and CR than did abnormally shaped spermatozoa (Table 1). At T24, abnormal spermatozoa from donors and patients had lower values of VSL, RF, BF and AMP than did the normally shaped cells (Table 1).

\section{Comparison between the movements of capacitated and uncapacitated sperm suspensions}

When the movement characteristics of the washed spermatozoa before and after incubation for $6 \mathrm{~h}$ were compared, a change in the swimming pattern could be clearly demonstrated for the morphologically normal spermatozoa of the donors. This change consisted of a significant increase in the AH values and a significant decrease in LIN, BF and CR (Table 1). There was also a tendency for an increase in the AMP values, but the difference did not reach statistical significance (Table 1). Although the VSL did not change, the decrease in LIN implies an increased curvilinear velocity. Since the beat frequency declined during this period, the apparently increased sperm vigour must be attributed to changes in flagellar beat shape. The frequency distributions of LIN, AH and CR values for the normal spermatozoa from donors, were different at T0 and T6 (Fig. 1). At the later time, there was a shift toward greater values of AH and smaller values of LIN and CR. The upper limit of AH increased and the lower limits for LIN and CR decreased. Analysis of AMP frequency distributions also shows a shift toward greater values at T6 (Fig. 1). Morphologically abnormal spermatozoa from the donors did not exhibit such changes in their movement characteristics after incubation for $6 \mathrm{~h}$ (Table 1 ).

Normally shaped spermatozoa from patients exhibited a significant decrease in VSL at T6, but had no significant changes in the values of LIN, RF, BF, AMP, AH and CR at T6 and T24 (Table 1). Analysis of the frequency distributions of the movement characteristic values of morphologically normal spermatozoa from patients at T0 and T6 did not reveal any changes at the later time 
Table 1. Movement characteristics of morphologically normal and abnormal spermatozoa from 10 fertile donors and 10 patients at three time points during in-vitro incubation under capacitating conditions

\begin{tabular}{|c|c|c|c|c|c|c|}
\hline \multirow[b]{2}{*}{ Movement characteristics } & \multicolumn{2}{|c|}{ T0 } & \multicolumn{2}{|r|}{ T6 } & \multicolumn{2}{|c|}{$\mathrm{T} 24$} \\
\hline & Normal & Abnormal & Normal & Abnormal & Normal & Abnormal \\
\hline \multicolumn{7}{|l|}{ Donors } \\
\hline Straight line velocity $(\mu \mathrm{m} / \mathrm{sec})$ & $\begin{array}{c}77 \cdot 8 \pm \\
7 \cdot 5\end{array}$ & $\begin{array}{c}66 \cdot 6 \pm \\
5 \cdot 7^{*}\end{array}$ & $\begin{array}{c}77 \cdot 9 \pm \\
8.2\end{array}$ & $\begin{array}{c}59 \cdot 7 \pm \\
6.0^{*}\end{array}$ & $\begin{array}{r}63 \cdot 2 \pm \\
7 \cdot 8+t\end{array}$ & $\begin{array}{r}47.6 \pm \\
6.9^{* \pm}+\end{array}$ \\
\hline Linearity & $\begin{array}{l}0.87 \pm \\
0.01\end{array}$ & $\begin{array}{l}0.87 \pm \\
0.01\end{array}$ & $\begin{array}{l}0.79 \pm \\
0.02 \dagger\end{array}$ & $\begin{array}{l}0.85 \pm \\
0.02^{*}\end{array}$ & $\begin{array}{l}0.85 \pm \\
0.02\end{array}$ & $\begin{array}{l}0.86 \pm \\
0.02\end{array}$ \\
\hline Rolling frequency (rolls/sec) & $\begin{array}{l}8 \cdot 1 \pm \\
0.8\end{array}$ & $\begin{array}{l}6 \cdot 6 \pm \\
0 \cdot 6^{*}\end{array}$ & $\begin{array}{l}7.9 \pm \\
0.8\end{array}$ & $\begin{array}{l}6.8 \pm \\
0.7^{*}\end{array}$ & $\begin{array}{l}7 \cdot 7 \pm \\
0.9\end{array}$ & $\begin{array}{l}5 \cdot 9 \pm \\
0 \cdot 9 *\end{array}$ \\
\hline Beat frequency (beats/sec) & $\begin{array}{c}24.2 \\
1.9\end{array}$ & $\begin{array}{c}20 \cdot 4 \pm \\
1 \cdot 3^{*}\end{array}$ & $\begin{array}{c}21 \cdot 5 \pm \\
2 \cdot 1 \dagger\end{array}$ & $\begin{array}{c}19.9 \pm \\
1.6^{*}\end{array}$ & $\begin{array}{r}19 \cdot 1 \pm \\
2.0 \dagger \ddagger\end{array}$ & $\begin{array}{r}16 \cdot 2 \pm \\
2 \cdot 0^{* t}\end{array}$ \\
\hline Amplitude $(\mu \mathrm{m})$ & $\begin{array}{l}5 \cdot 7 \pm \\
0.3\end{array}$ & $\begin{array}{l}5 \cdot 3 \pm \\
0 \cdot 3^{*}\end{array}$ & $\begin{array}{l}6 \cdot 1 \pm \\
0.2\end{array}$ & $\begin{array}{l}5 \cdot 4 \pm \\
0.3^{*}\end{array}$ & $\begin{array}{l}5 \cdot 8 \pm \\
0.2\end{array}$ & $\begin{array}{l}5 \cdot 0 \pm \\
0 \cdot 3^{*}\end{array}$ \\
\hline Amplitude lateral head displacement $(\mu \mathrm{m})$ & $\begin{array}{l}4 \cdot 4 \pm \\
0 \cdot 2\end{array}$ & $\begin{array}{l}4 \cdot 4 \pm \\
0 \cdot 2\end{array}$ & $\begin{array}{c}5.5 \pm \\
0.4 \dagger\end{array}$ & $\begin{array}{l}4 \cdot 5 \pm \\
0 \cdot 3^{*}\end{array}$ & nd & nd \\
\hline Curvature ratio & $\begin{array}{l}0.83 \pm \\
0.01\end{array}$ & $\begin{array}{l}0.83 \pm \\
0.01\end{array}$ & $\begin{array}{l}0.70 \pm \\
0.02 \dagger\end{array}$ & $\begin{array}{l}0.81 \pm \\
0.01^{*}\end{array}$ & nd & nd \\
\hline \multicolumn{7}{|l|}{ Patients } \\
\hline Straight line velocity $(\mu \mathrm{m} / \mathrm{sec})$ & $\begin{array}{c}77 \cdot 0 \pm \\
5 \cdot 4\end{array}$ & $\begin{array}{c}55 \cdot 3 \pm \\
7 \cdot 3^{*}\end{array}$ & $\begin{array}{c}68 \cdot 8 \pm \\
3 \cdot 7 \dagger\end{array}$ & $\begin{array}{c}56 \cdot 4 \pm \\
4 \cdot 2^{*}\end{array}$ & $\begin{array}{r}52 \cdot 7 \pm \\
6.4 \dagger \ddagger\end{array}$ & $\begin{array}{r}44 \cdot 3 \pm \\
4 \cdot 8^{* \pm}\end{array}$ \\
\hline Linearity & $\begin{array}{l}0.84 \pm \\
0.02\end{array}$ & $\begin{array}{l}0.85 \pm \\
0.02\end{array}$ & $\begin{array}{l}0.84 \pm \\
0.02\end{array}$ & $\begin{array}{l}0.86 \pm \\
0.02\end{array}$ & $\begin{array}{l}0.86 \pm \\
0.01\end{array}$ & $\begin{array}{l}0.88 \pm \\
0.01\end{array}$ \\
\hline Rolling frequency (rolls/sec) & $\begin{array}{l}7.7 \pm \\
1.1\end{array}$ & $\begin{array}{l}5 \cdot 7 \pm \\
1 \cdot 0^{*}\end{array}$ & $\begin{array}{l}7.9 \pm \\
0.6\end{array}$ & $\begin{array}{c}6.7 \pm \\
0.8^{*} \dagger\end{array}$ & $\begin{array}{l}6.9 \pm \\
1 \cdot 2\end{array}$ & $\begin{array}{l}5 \cdot 8 \pm \\
0.9^{*} \ddagger\end{array}$ \\
\hline Beat frequency (beats/sec) & $\frac{21.4}{1.7} \pm$ & $\begin{array}{c}16 \cdot 8 \pm \\
2 \cdot 2^{*}\end{array}$ & $\begin{array}{c}20 \cdot 5 \pm \\
1.1\end{array}$ & $\begin{array}{c}18 \cdot 1 \pm \\
1 \cdot 1^{*}\end{array}$ & $\begin{array}{r}17 \cdot 8 \pm \\
2 \cdot 2 \dagger \ddagger\end{array}$ & $\begin{array}{r}15.8 \pm \\
1.8^{*} \ddagger\end{array}$ \\
\hline Amplitude $(\mu \mathrm{m})$ & $\begin{array}{l}5 \cdot 7 \pm \\
0.4\end{array}$ & $\begin{array}{l}4 \cdot 9 \pm \\
0 \cdot 3^{*}\end{array}$ & $\begin{array}{l}5 \cdot 7 \pm \\
0.4\end{array}$ & $\begin{array}{l}5.4 \pm \\
0.3 \dagger\end{array}$ & $\begin{array}{l}5 \cdot 1 \pm \\
0 \cdot 4\end{array}$ & $\begin{array}{l}4 \cdot 6 \pm \\
0.3^{*} t\end{array}$ \\
\hline Amplitude lateral head displacement $(\mu \mathrm{m})$ & $\begin{array}{l}4 \cdot 0 \pm \\
0 \cdot 2\end{array}$ & $\begin{array}{l}3.7 \pm \\
0.5\end{array}$ & $\begin{array}{l}4 \cdot 2 \pm \\
0 \cdot 3\end{array}$ & $\begin{array}{l}4 \cdot 1 \pm \\
0 \cdot 3\end{array}$ & nd & nd \\
\hline Curvature ratio & $\begin{array}{l}0.86 \pm \\
0.01\end{array}$ & $\begin{array}{l}0.88 \pm \\
0.01\end{array}$ & $\begin{array}{l}0.83 \pm \\
0.01\end{array}$ & $\begin{array}{l}0.85 \pm \\
0.01\end{array}$ & nd & nd \\
\hline
\end{tabular}

Values are mean \pm s.e.m. of individual ejaculate means for 25 normal and 25 abnormal spermatozoa. nd $=$ no data. *Significantly different from corresponding value for normal spermatozoa $(P<0 \cdot 01)$.

tSignificantly different from corresponding value at T0 $(P<0.025)$.

$\$$ Significantly different from corresponding value at T6 $(P<0.01)$.

(data not shown). The abnormal spermatozoa from patients showed a significant increase in RF and AMP at T6, but no other change in movement characteristics (Table 1). The changes in the patterns of sperm motion seen in normal donor spermatozoa under capacitation conditions were not observed in the sperm suspensions from patients.

After incubation for $24 \mathrm{~h}$, there was a decrease in the VSL and flagellar BF of the morphologically normal and abnormal spermatozoa, as compared to the $\mathrm{T} 0$ and $\mathrm{T} 6$ values (Table 1). This was true for donors and patients and probably reflected sperm senescence.

\section{Sperm penetration assay}

Spermatozoa from 5 of the 10 patients were unable to penetrate the zona-free hamster oocytes in these experiments. The percentages of oocytes penetrated by spermatozoa of the other patients 


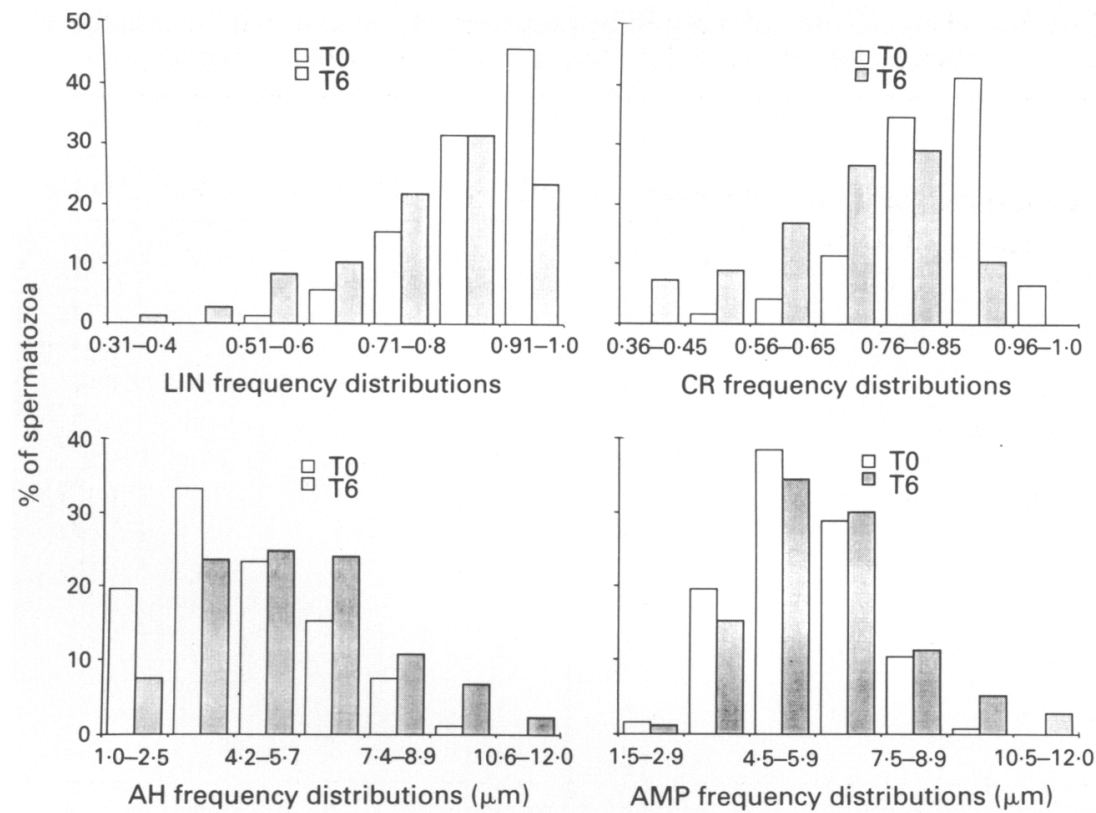

Fig. 1. Pooled frequency distributions of the movement characteristics (linearity (LIN), amplitude of lateral head displacement (AH), curvature ratio (CR), and flagellar amplitude (AMP)) of the morphologically normal spermatozoa from fertile donors at $\mathrm{T} 0$ and at $\mathrm{T} 6$. Values are for 25 spermatozoa at each time from each of 10 normal donors.

Table 2. Sperm penetration of zona-free hamster oocytes

\begin{tabular}{|c|c|c|c|c|c|c|c|}
\hline \multirow{2}{*}{$\frac{\text { Patient }}{1}$} & \multicolumn{2}{|c|}{$\%$ Penetration ${ }^{*}$} & \multirow{2}{*}{$\frac{\begin{array}{c}\text { Spermatozoa/ } \\
\text { egg } \dagger\end{array}}{-}$} & \multirow{2}{*}{$\frac{\text { Donor }}{11}$} & \multicolumn{2}{|c|}{$\%$ Penetration $*$} & \multirow{2}{*}{$\begin{array}{c}\begin{array}{c}\text { Spermatozoa/ } \\
\text { egg } \dagger\end{array} \\
3.8\end{array}$} \\
\hline & 0 & $(0 / 52)$ & & & 100 & $(50 / 50)$ & \\
\hline 2 & 0 & $(0 / 50)$ & - & 12 & 96 & $(44 / 46)$ & $2 \cdot 6$ \\
\hline 3 & 0 & $(0 / 45)$ & - & 13 & 55 & $(18 / 33)$ & $1 \cdot 1$ \\
\hline 4 & 13 & $(4 / 30)$ & 1.0 & 14 & 50 & $(25 / 50)$ & 1.4 \\
\hline 5 & 20 & $(8 / 40)$ & $1 \cdot 25$ & 15 & 72 & $(26 / 36)$ & 1.6 \\
\hline 6 & 0 & $(0 / 43)$ & - & 16 & 67 & $(28 / 42)$ & 1.4 \\
\hline 7 & 0 & $(0 / 43)$ & - & 17 & 77 & $(30 / 39)$ & $1 \cdot 7$ \\
\hline 8 & 30 & $(10 / 33)$ & $1 \cdot 1$ & 18 & 55 & $(21 / 38)$ & $1 \cdot 3$ \\
\hline 9 & 31 & $(9 / 29)$ & 1.0 & 19 & 59 & $(19 / 32)$ & 1.2 \\
\hline 10 & 26 & $(9 / 35)$ & 1.0 & 20 & 64 & $(27 / 42)$ & 1.4 \\
\hline Mean \pm s.e.m. & \multicolumn{2}{|c|}{$12 \cdot 0 \pm 4 \cdot 3$} & $1 \cdot 1 \pm 0 \cdot 5$ & \multicolumn{3}{|c|}{$69 \cdot 5 \pm 5 \cdot 4$} & $1.75 \pm 0.3$ \\
\hline
\end{tabular}

*In parentheses is the number of penetrated oocytes in relation to the total number of oocytes.

$\dagger$ Number of decondensed sperm heads with associated tails in the cytoplasm per egg.

were relatively low ( $13 \%$ to $31 \%)$, as was the number of swollen sperm heads per oocyte (Table 2 ). In contrast, the spermatozoa of donors were always successful in penetrating the hamster oocytes and there was a mean of 1.75 spermatozoa per oocyte (Table 2 ).

When we compared the movement characteristics of spermatozoa from the donors and patients at T24 with the percentage of the zona-free hamster oocytes penetrated, positive correlations were only found when the morphologically normal spermatozoa were considered. For the donors, the 


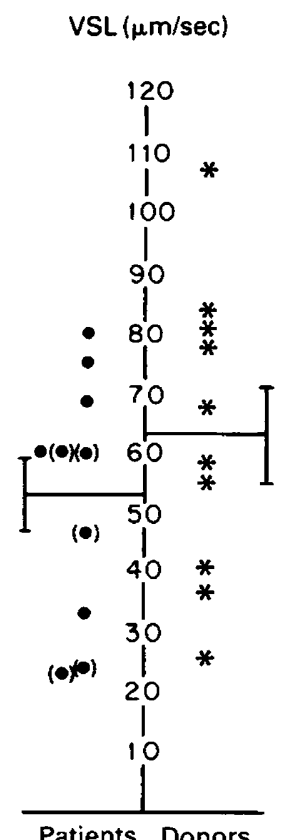

BF (beats/sec)

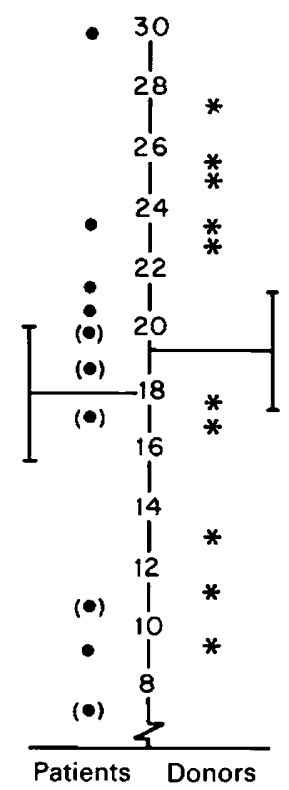

RF (rolls/sec)

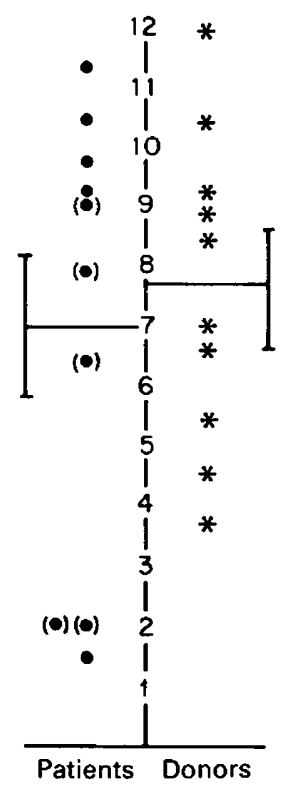

Fig. 2. Movement characteristics of the morphologically normal spermatozoa from fertile donors and patients at T24 in relation to the result of the zona-free hamster oocyte penetration test. Values in parentheses $(\bullet)$ denote the patients from which spermatozoa failed to penetrate the hamster oocytes. The horizontal line with a perpendicular line at its end represents the mean \pm s.e.m.

only significant correlation was with a measure of beat shape (AMP) at T6 (correlation coefficient $0.72, P<0.05$ ). In contrast, success of the patients' spermatozoa in penetrating hamster oocytes was correlated with movement characteristics reflecting sperm vigour (i.e. BF, RF, VSL) at T0, T6 and T24. Correlation coefficients of $\mathrm{BF}$ at $\mathrm{T} 0$ and of $\mathrm{BF}$ and $\mathrm{RF}$ at $\mathrm{T} 6$ were $0 \cdot 73,0.62$, and 0.65 , respectively $(P<0.05)$. At T24, the correlation coefficients for VSL, RF and flagellar BF were $0 \cdot 77$, 0.76 , and 0.71 , respectively $(P<0.05)$. Analysis of the regression lines showed that all the slopes were different from zero $(P<0.01)$. Among the patients there was a clear distinction between the vigour of sperm suspensions that penetrated zona-free hamster oocytes and those that failed to penetrate (Fig. 2). However, some of the sperm suspensions that failed to penetrate the zonafree hamster oocytes had movement characteristics that were similar to those of donor sperm suspensions (Fig. 2).

\section{Discussion}

Previous studies from our laboratory have shown that there is a close relationship between sperm head morphology and motility in semen (Overstreet et al., 1981; Katz et al., 1982; Morales et al., 1988). We have reported that a greater percentage of morphologically normal spermatozoa (whether they are present in the semen of fertile donors or patients) are motile, and that they are more vigorous than abnormally shaped cells (Morales et al., 1988). In the present study the movement characteristics of morphologically normal and abnormal spermatozoa were evaluated during in-vitro capacitation. The conditions used in our incubation protocol were such that it is likely that some of the spermatozoa in the suspensions became capacitated after $6 \mathrm{~h}$ (Perreault \& Rogers, 1982; Gould et al., 1983). When the movement characteristics of morphologically normal and 
(a)

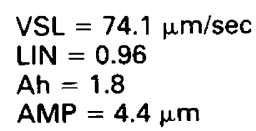

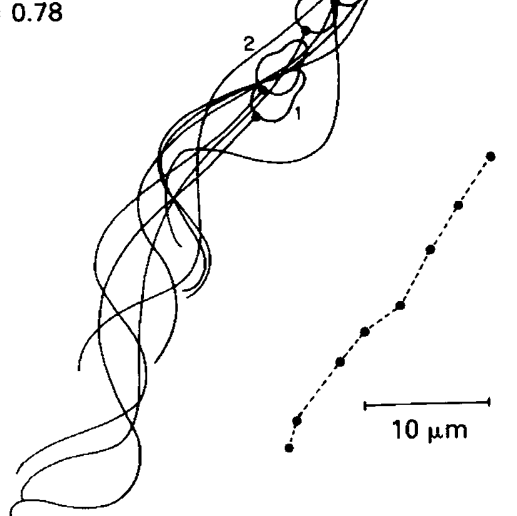

Fig. 3. Movement characteristics (see text for abbreviations) and swimming trajectories of a morphologically normal spermatozoon from a fertile donor at (a) T0 and (b) T6. Consecutive tracings are (a) $0.032 \mathrm{sec}$ or (b) $0.049 \mathrm{sec}$ apart. Points connected by broken lines represent the sequential position of the sperm head.

abnormal spermatozoa were compared after removal of the seminal plasma, normal spermatozoa swam faster and exhibited higher values of RF, BF and AMP than did the abnormal spermatozoa throughout the entire incubation period. This difference between morphological types was true for donors and patients. We have suggested previously that spermatozoa from donors and patients may not be equivalent in their physiology, even if they both fit our definition of normal head morphology (Overstreet et al., 1981; Morales et al., 1988). The present findings support this hypothesis.

In the current study, a set of changes in the characteristics of sperm movement, analogous to the hyperactivated motility reported for other species, became apparent after incubation for $6 \mathrm{~h}$. These changes were only significant in the population of normal spermatozoa from donors, and could be appreciated subjectively as a difference in the pattern of sperm motion. Sperm swimming trajectories were less straight, and there was a greater degree of lateral head displacement about the average path. Changes in the flagellar beat included a higher degree of bending in the proximal flagellum and an increase in beat amplitude. During the same incubation interval, the flagellar BF decreased. This decrease in BF, accompanying the increase in flagellar bending, is analogous to flagellar changes recorded for other mammalian spermatozoa during hyperactivation. The reduction of $\mathbf{B F}$ does not imply that the spermatozoa were any less vigorous (Katz et al., 1978). In fact, their curvilinear velocity increased. The increased sperm vigour must therefore be attributed to the increased flagellar bending, a hallmark of hyperactivation. These changes were not seen in all the spermatozoa within an ejaculate but in a subset, as evidenced by the frequency distributions. In Fig. 3, the sequential positions of the sperm head and flagellum, with the respective movement indicators, are given for a morphologically normal spermatozoon from a fertile donor at T0 and T6. These patterns illustrate the motion that, under our incubation conditions, may typify human sperm hyperactivation in vitro.

Other investigators have described hyperactivated motility in human sperm suspensions. Burkman (1984) observed a higher incidence of spermatozoa with wide-amplitude, low-frequency 
flagellar beating following in-vitro incubation in comparison with semen. Mortimer et al. (1984) reported an increase in the percentage of spermatozoa exhibiting high values of $\mathrm{AH}$ and nonprogressive motility after $5 \mathrm{~h}$ of incubation. Although both studies presented data on sperm swimming trajectories, the flagellar beat characteristics responsible for those trajectories were not measured. The use of a high-speed video camera in the present study enabled us to make direct and accurate measurements of the flagellar beat shape. One of these indicators, the flagellar CR, revealed that capacitated human spermatozoa exhibit a high degree of curvature in the proximal flagellum. Other studies have revealed similar changes during hyperactivation of spermatozoa from guinea-pigs (Katz et al., 1978; Katz \& Yanagimachi, 1981), hamsters (Katz et al., 1978, 1986; Suarez et al., 1984), mice (Fraser, 1977), monkeys (Behboodi et al., 1987), rabbits (Johnson et al., 1981; Suarez et al., 1983) and rams (Cummins, 1982).

The fact that we were unable to demonstrate a systematic shift to hyperactivated movement in sperm suspensions from patients may indicate failure or inefficiency of capacitation, and therefore could be relevant to the cause of their infertile marriage. Hyperactivated motility may be involved in the final ascent of spermatozoa to the site of fertilization in the tubal ampulla, and/or in the generation of flagellar thrust required for cumulus and zona pellucida penetration (Yanagimachi, 1981; Katz et al., 1987). If hyperactivation is a marker for sperm capacitation, then capacitation was relatively less efficient for the spermatozoa of the patients under the conditions of these experiments. When zona-free hamster oocytes were added to these sperm suspensions at T24, fusion with the oocytes was observed in only 5 of the 10 cases. Although we do not know the reasons for the failures of sperm penetration in these experiments, the apparent differences in movement characteristics between the successful and unsuccessful sperm suspensions may reflect the general health of the spermatozoa rather than a specific alteration in function related to gamete interaction. Thus, the indicators of sperm movement that reflect the vigour of flagellar activity, rather than the pattern changes of hyperactivation, were those that correlated best with hamster oocyte penetration. Our inability to correlate directly the movement typifying hyperactivation with the success of oocyte penetration may be related, in part, to the excessive sperm numbers in suspension relative to the small number of spermatozoa that interact with oocytes. Moreover, hyperactivated movement was detected at T6, but not at T24 when the sperm penetration assay was performed. A clearer relationship between gamete fusion events and sperm movement might have been revealed if the oocytes had been added to the sperm suspensions at the eartier time point.

We do not fully understand the kinetics of human sperm capacitation. Perreault \& Rogers (1982) reported that the kinetics of capacitation may differ among individuals, but that the minimum capacitation time seems to be very short. Under appropriate conditions, $<2 \mathrm{~h}$ may be sufficient for human sperm capacitation, the acrosome reaction, and subsequent zona pellucida penetration (McMaster et al., 1978; Overstreet et al., 1980). In the present study we observed that, after 3 cycles of washing and centrifugation, some morphologically normal spermatozoa from donors exhibited values of AH, CR and LIN that were similar to those observed in the larger sperm population after incubation for $6 \mathrm{~h}$. This may be an indication that some spermatozoa became capacitated during this short interval. Even at longer intervals it is not possible to determine specifically what fraction of spermatozoa became capacitated. The overall proportion may be small. The occurrence of the acrosome reaction, another hallmark of capacitation, appears to take place in a relatively small fraction of human spermatozoa exposed to capacitation conditions in vitro (Plachot et al., 1984; Mallet et al., 1985; Nagae et al., 1986).

The morphologically normal spermatozoa from patients did not show the same type of changes during incubation as did the normal spermatozoa from donors. This distinction suggests that these populations either lack the ability to exhibit hyperactivation under conditions that promote this change in functional spermatozoa, or that hyperactivation is undergone by a much smaller segment of the sperm populations. Burkman (1984) reported that the incidence of hyperactivated motility in her patient population was less than half the rate for the fertile donors. In our study there were some normal spermatozoa from the patients that exhibited values of AH, CR, LIN and AMP at T6 
which approximated those exhibited by normal spermatozoa from the donors. Although we believe that the spermatozoon depicted in Fig. 3 was hyperactivated, inspection of the histograms in Fig. 1 reveals substantial heterogeneity of movement characteristics within the population of sperm cells. We still lack absolute criteria that define an individual human spermatozoon as being hyperactivated and our study contributes to the objective definition of this phenomenon. More detailed analyses of hyperactivation will be necessary before we can characterize human sperm populations in terms of the percentage hyperactivated cells.

We thank Dr Nicholas L. Cross and Dr Steven J. Samuels for helpful suggestions. This research was supported by NIH grants HD 15149 (J.W.O.) and ES03614 (D.F.K.)

\section{References}

Aitken, R.J., Best, F.S.M., Richardson, D.W., Djahanbakhch, O. \& Lees, M.M. (1982) The correlates of fertilizing capacity in normal fertile men. Fert. Steril. 38, 68-76.

Aitken, R.J., Best, F.S.M., Warner, P. \& Templeton, A. (1984) A prospective study of the relationship between semen quality and fertility in cases of unexplained infertility. J. Androl. 5, 297-303.

Aitken, R.J., Sutton, M., Warner, P. \& Richardson, D.W. (1985) Relationship between the movement characteristics of human spermatozoa and their ability to penetrate cervical mucus and zona-free hamster oocytes. J. Reprod. Fert. 73, 441-449.

Behboodi, E., Katz, D.F., Overstreet, J.W. \& Hendrickx, A. (1987) Movement characteristics of Cynomolgus monkey sperm recovered from the cervix and uterus. J. Androl. 8, 42P, Abstr.

Biggers, J.D., Whitten, W.K. \& Whittingham, D.G. (1971) The culture of mouse embryos in vitro. In Methods in Mammalian Embryology, pp. 86-116. Ed J. C. Daniels. W. H. Freeman, San Francisco.

Burkman, L.J. (1984) Characterization of hyperactivated motility by human spermatozoa during capacitation: comparison of fertile and oligozoospermic sperm populations. Archs Androl. 13, 153-165.

Cooper, G.W., Overstreet, J.W. \& Katz, D.F. (1979) The motility of rabbit spermatozoa recovered from the female reproductive tract. Gamete Res. 2, 35-42.

Cummins, J.M. (1982) Hyperactivated motility patterns of ram spermatozoa recovered from the oviducts of mated ewes. Gamete Res. 6, 53-63.

David, G., Serres, C. \& Jouannet, P. (1981) Kinematics of human spermatozoa. Gamete Res. 4, 83-95.

Fraser, L.R. (1977) Motility patterns in mouse spermatozoa before and after capacitation. J. exp. Zool. 202, 439-444.

Gould, J.E., Overstreet, J.W., Yanagimachi, H., Yanagimachi, R., Katz, D.F. \& Hanson, F.W. (1983) What functions of the sperm cell are measured by in vitro fertilization of zona-free hamster eggs? Fert. Steril. 40, 344-352.

Johnson, L.L., Katz, D.F. \& Overstreet, J.W. (1981) The movement characteristics of rabbit spermatozoa before and after activation. Gamete Res. 4, $275-282$.

Katz, D.F. \& Yanagimachi, R. (1980) Movement characteristics of hamster spermatozoa within the oviduct. Biol. Reprod. 22, 759-764.
Katz, D.F. \& Yanagimachi, R. (1981) Movement characteristics of hamster and guinea pig spermatozoa upon attachment to the zona pellucida. Biol. Reprod. 25, 785-791.

Katz, D.F., Yanagimachi, R. \& Dresdner, R.D. (1978) Movement characteristics and power output of guinea pig and hamster spermatozoa in relation to activation. J. Reprod. Fert. 52, 167-172.

Katz, D.F. \& Overstreet, J.W. (1980) Mammalian sperm movement in the secretions of the male and female genital tracts. In Testicular Development, Structure and Function, pp. 481-489. Eds A. Steinberger \& E. Steinberger. Raven Press, New York.

Katz, D.F., Diel, L. \& Overstreet, J.W. (1982) Differences in the movement of morphologically normal and abnormal human seminal spermatozoa. Biol. Reprod. 26, 566-570.

Katz, D.F., Cherr, G.N. \& Lambert, H. (1986) The evolution of hamster sperm motility during capacitation and interaction with the ovum vestments in vitro. Gamete Res. 14, 333-346.

Katz, D.F., Drobnis, E.Z., Baltz, J., Cherr, G.N., Yudin, A.I., Core, R.A. \& Chen, L.Y. (1987) The biophysics of sperm penetration of the cumulus and zona pellucida. In New Horizons in Sperm Cell Research, pp. 13-26. Ed. H. Mohri. Japan Sci. Soc. Press, Tokyo.

McMaster, R., Yanagimachi, R. \& Lopata, A. (1978) Penetration of human eggs by human spermatozoa in vitro. Biol. Reprod. 19, 212-216.

Mallet, P.J., Stock, C.E. \& Fraser, L.R. (1985) Acrosome loss in human sperm incubated in vitro under capacitating conditions. Int. J. Androl. 8, 357-366.

Morales, P., Katz, D.F., Overstreet, J.W., Samuels, S.J. \& Chang, R.J. (1988) The relationship between the motility and morphology of spermatozoa in human semen. J. Androl. (in press).

Mortimer, D., Courtot, A.M., Giovangrandi, Y., Jeulin, C. \& David, G. (1984) Human sperm motility after migration into, and incubation in, synthetic media. Gamete Res. 9, 131-144.

Nagae, T., Yanagimachi, R., Srivastava, P.N. \& Yanagimachi, H. (1986) Acrosome reaction in human spermatozoa. Fert. Steril. 45, 701-707.

Okada, A., Kato, M., Fujio, K., Miura, K., Nakayama, K. \& Shirai, M. (1985) Hyperactivation of human spermatozoa: measurement of motility before and after incubation. Andrologia 17, 583-586. 
Okada, A., Kato, M., Fujio, K., Miura, K., Nakayama, K. \& Shirai, M. (1986) Hyperactivation of human sperm and zona-free hamster egg penetration assay. $Z$. mikrosk. Anat. Forsch., Leipzig 100, 233-240.

Overstreet, J.W., Yanagimachi, R., Katz, D.F., Hayashi, K. \& Hanson, F.W. (1980) Penetration of human spermatozoa into the human zona pellucida and the zona-free hamster eggs: a study of fertile donors and infertile patients. Fert. Steril. 33, 534-542.

Overstreet, J.W., Price, M.J., Blazak, W.F., Lewis, E.L. \& Katz, D.F. (1981) Simultaneous assessment of human sperm motility and morphology by videomicrography. J. Urol. 126, 357-360.

Perreault, S.D. \& Rogers, B.J. (1982) Capacitation pattern of human spermatozoa. Fert. Steril. 38, $258-260$.

Plachot, M., Mandelbaum, J. \& Junca, A.M. (1984) Acrosome reaction of human sperm used for in vitro fertilization. Fert. Steril. 42, 418-423.

Suarez, S.S., Katz, D.F. \& Overstreet, J.W. (1983) Movement characteristics and acrosomal status of rabbit spermatozoa recovered at the site and time of fertilization. Biol. Reprod. 29, 1277-1287.
Suarez, S.S., Katz, D.F. \& Meizel, S. (1984) Changes in motility that accompany the acrosome reaction in hyperactivated hamster spermatozoa. Gamete Res. 10, 253-265.

Yanagimachi, R. (1970) The movement of golden hamster spermatozoa before and after capacitation. J. Reprod. Fert. 23, 193-196.

Yanagimachi, R. (1981) Mechanisms of fertilization in mammals. In Fertilization and Embryonic Development In Vitro, pp. 81-181. Eds L. Mastroianni \& J. D. Biggers. Plenum Publishing Corporation, New York.

Yanagimachi, R., Yanagimachi, H. \& Rogers, B.J. (1976) The use of zona-free animal ova as a test-system for the assessment of the fertilizing capacity of human spermatozoa. Biol. Reprod. 15, 471-476.

Yanagimachi, R., Lopata, A., Odom, C.B., Bronson, R.A., Mahi, C.A. \& Nicolson, G.L. (1979) Retention of biologic characteristics of zona pellucida in highly concentrated salt solution: the use of salt-stored eggs for assessing the fertilizing capacity of spermatozoa. Fert. Steril. 31, 562-574.

Received 7 July 1987 